Investigaciones Fenomenológicas, vol. Monográfico 4/I (2013): Razón y vida, 79-92. e-ISSN: $1885-1088$

\title{
LA RAISON EN TANT QUE PRATIQUE SUBJECTIVE.
}

\section{LA RAZÓn COMO PRÁctica SUBJetiva. PARA UNA RECONSTRUCCIÓn DEL SIGNIFICADO DE LAS CONDUCTAS SOCIOCULTURALES.}

\author{
Ion Copoeru \\ Centrul de Cercetari Aplicate in Fenomenologie (CECAF)/ \\ Babeş-Bolyai University Cluj-Napoca, Rumania \\ copoeru@hotmail.com
}

\begin{abstract}
Résumé: Le but de ce papier est d'argumenter en la faveur de l'idée qu'il est possible non seulement de donner une place particulière à la raison dans notre vie et dans la société, mais aussi d'offrir un cadre rationnel intégratif, dans lequel les fins et buts humains trouvent leur expression rationnelle.

Le texte comporte trois parties. La première décrit l'approche pratique-herméneutique d'Alfred Schutz à l'égard de la loi et de la normativité, tout en faisant place à une pratique subjective de la raison (phénoménologiquement dévoilée). La deuxième se propose de dévoiler, à travers la description de la connaissance dans le monde de la vie ordinaire, une forme de rationalité qui nous offre des voies pratiques mais consistantes de prendre une décision. En fin de compte, la troisième vise la reconstruction pratique-herméneutique de la loi, afin d'intégrer dans le noyau du raisonnement légal (normatif) des références à des fins et buts sans porter préjudice à l'objectivité du raisonnement lui-même.

L'étude veut apporter une contribution au problème de la dédifférenciation et du positivisme pratique dans le monde moderne.
\end{abstract}

Mots clef: Alfred Schutz, rationalité, loi, pratique, buts.
Resumen: El objetivo de este trabajo es argumentar a favor de la idea de que es posible, no sólo dar un lugar especial a la razón en nuestras vidas y en la sociedad, sino también proporcionar un marco racional integrador en el que los propósitos y objetivos humanos encuentren expresión racional.

El texto consta de tres partes. La primera describe el enfoque práctico-hermenéutico de Alfred Schutz en relación con la ley y la normatividad, abriendo un espacio a la práctica subjetiva de la razón (desvelada fenomenológicamente). La segunda propone dar a conocer, a través de la descripción del conocimiento en el mundo de la vida ordinaria, una forma de racionalidad que nos ofrece vías prácticas pero consistentes para tomar decisiones. Por último, la tercera es la práctica hermenéutica de reconstrucción de la ley, para incluir en el núcleo del razonamiento jurídico (normativo) referencias a fines y objetivos sin menoscabar la objetividad del razonamiento en sí.

El estudio tiene como objetivo contribuir al problema de la desdiferenciación y del positivismo práctico en el mundo moderno.

Palabras clave: Alfred Schutz, racionalidad, ley, práctica, objetivos. 


\section{INTRODUCTION}

Dans la société moderne, le rôle de la connaissance formelle et des experts professionnels s'accroît constamment, ce qui rend la connaissance déterminante, devenant ainsi un élément clé de la gestion des questions sociales. C'est la raison pour laquelle l'intérêt des philosophes et des scientifiques du social s'est déplacé, dans les derniers temps, vers les politiques de la connaissance comment les idées sont créées, utilisées et disséminées. En conséquence, il semble que la meilleure façon d'améliorer le résultat des activités de connaissance consiste dans l'amélioration des politiques de la connaissance, dans la mise à l'épreuve des institutions et de leur manière de constitution afin qu'elles soient en mesure de livrer de la connaissance qui corresponde mieux aux besoin perçus de la société, et de remplir les fins et les intérêts d'autant de membres que possible.

Pourtant, cette façon d'aborder les choses, inaugurée dans la première partie du dernier siècle par la - ainsi nommée - "sociologie de la connais-sance", a semblé encourager le relativisme et détruire les fondations épistémiques de notre vision du monde et de notre manière rationnelle de comprendre le monde.

Dans mon opinion, tel n'est pas nécesseraiment le cas et le but général de ce papier est d'argumenter en la faveur d'un type d'approche spécifique à la "sociologie de la connaissance", en montrant qu'il est possible qu'elle nous conduise, non seulement à donner une place particulière à la raison dans notre vie et dans la société, mais aussi à offrir un cadre rationnel intégratif, dans lequel les fins et buts humains trouvent leur expression rationnelle.

\section{L'APPROCHE PRATIQUE-HERMENEUTIQUE D'ALFRED}

SCHUTZ A L'EGARD DE LA LOI ET DE LA NORMATIVITE

Le terme de "normativité" est utilisé par Alfred Schutz seulement en connexion avec la théorie de I'action sociale de Talcott Parsons (Schutz, 1978 and 1996). Même s'il n'appartient pas au vocabulaire propre de Schutz, le terme est hautement significatif pour l'originalité de l'approche schutzienne du monde 
social et de la loi. En couvrant tous les phénomènes qui présupposent une référence à ce qu'on devrait faire, la normativité nous offre un point de vue privilégié, où les questions éthiques gagnent en pertinence. Mon hypothèse est que cela ouvre une voie d'accès vers une approche phénoménologique de la loi et des pratiques légales dans une société moderne juridisée. Dans cette perspective, la tâche du philosophe de la loi est de la décrire de telle manière que l'entier système normatif de la société soit rendu à la fois perméable tant aux actions de l'individu, qu'aux actions collectives, et susceptible de se changer en fonction d'une certaine dimension historique des principes de justice. Afin d'y réussir, cette description phénoménologique doit être doublée d'une approche pratique-herméneutique. Alors que les herméneutiques légales se rapportaient au noyau de la pratique légale, c'est-à-dire à l'interprétation des textes légaux en tant que sens, plus ou moins indéterminé (Kress, 1992, 200-218), les visées des herméneutiques légales, en ce qui concerne la pratique, ont été modestes et, au fond, prisonnières d'un point de vue extérieur à la pratique de la loi. Or, le point de vue de la pratique est néanmoins obligatoire pour toute perspective herméneutique, et le but de toute activité interprétative ou théorétique est d'éclaircir à nouveau frais la pratique dans le domaine respectif. En conséquence, s'occuper de l'interprétation des textes légaux ne peut pas exclure I'interrogation des textes en tant que contenant une validité d'un genre spécial, exprimée dans un contexte vécu éminemment pratique.

Une herméneutique légale, qui est soutenue par une pratique subjective de la raison (phénoménologiquement dévoilée) est à même d'incarner le point de vue interne de la loi et d'illuminer ce qui est inhérent dans l'acte de jugement (en tant que, à la fois, interprétation des textes légaux et raisonnement dans des termes légaux). Afin de montrer cela, je vais m'occuper dans la première section de ce papier de la question de la raison et de la subjectivité, plus précisément de la question du raisonnement en tant que pratique subjectivephénoménologique. Dans la deuxième section, je vais me concentrer sur la tâche de reconstruire la loi d'un point de vue pratique-herméneutique.

\section{COORDONNEES D'UNE PRATIQUE DE LA RATIONALITE}


Schutz s'abstient de parler de fins et valeurs dernières. Cette attitude pourrait être l'expression de la prudence à l'égard de termes philosophiques insufisamment clarifiés et d'une sorte d'auto-limitation méthodologique. Pourtant, cela ne signifie pas que Schutz n'a pas continué d'examiner la nature des valeurs. Une analyse plus profonde montre que cette attitude restrictive est principalement la conséquence de l'adoption et du maintien d'un "point de vue subjectif". En parlant de "valeurs normatives" de Parsons, qui dans le vocabulaire schutzien représentent "un système de motifs", Schutz insiste qu'elle sont "avant tout une fonction de la vie de l'esprit ( $m i n d$ ) humain dans le temps, qui veut dire, dans la "durée", pour utiliser un terme bergsonien. Toute description vraiment subjective doit se référer à ce fait, qui d'un autre côté est difficilement compatible avec la conception des valeurs dernières ou des fins dernières (ultimate), ou avec une normativité à laquelle on peut se conformer seulement d'une manière temporaire (Schutz, 1978, 36). Le niveau dernier, s'il y en a un, est pour Schutz "le déplacement continu de l'intérêt, de la pertinence et de l'attention" (Ibid.), ouvert à une description phénoménologique.

Schutz rejete la conception de la normativité basée sur l'assomption qu'il y a un acteur, d'un côté, et une valeur objective ou objectifiée, d'un autre. Par conséquent, il trouve que le terme "volonté" (volition), qui implique "un effort de la part de l'acteur d'adapter son rôle en tant qu'agent au modèle de la valeur téléologique" (Schutz, 1978, 27), soit insuffisant pour décrire I'action sociale, et qu'aussi le nom "volontarisme" (voluntaristic), par lequel Parsons indique sa théorie, soit plutôt "étrange" (Ibid.).

Contrairement à ceux qui avaient suivi une voie néoidéaliste ou néoréaliste et avaient objectifié le sens, Schutz a renoncé par un geste philosophique radical, qui est passé innaperçu, à toute théorie des fondements transcendants d'un ordre préformée. (Schutz 1952, 224). Schutz a adopté un point de vue anti-essentialiste et, en revanche, son attention a été retenue par "la nature des relations qui, conformément à Weber, sont caractérisées par la 'probabilité de I'apparition répétée du comportement qui correspond à son sens subjectif'" (Schutz 1932, 39). Ainsi, la compréhension du monde social objectif dépend de sa potentielle accessibilité à mon expérience directe. Mais l'accès à mon expérience directe n'est pas un but en lui-même. Le motif pragmatique devient important: "Ici, aussi, agir (acting) 'est mesuré en fonction de' son succès ou in- 
succès ; ici, aussi, la validité du schème interprétatif du type de réalité est mise à l'épreuve par sa finalité" (Schutz 1928, 76).

En mettant entre parenthèses la question métaphysique à l'égard de la normativité, Schutz a fait place à la question phénoménologique des contextes dans lesquels les gens ordinaires font l'expérience des impératifs dans I'interaction entre l'appartenence subjective et objective à un groupe et entre les systèmes de pertinence imposés et typifications (Schutz 1964a, 265). À part I'interprétation objective de l'appartenence à un groupe, Schutz a en vue un type de normativité qui s'exprime dans la forme d'un échange réciproque de perspectives. Nous arrivons ainsi à la conséquence que le concept métaphysique de normativité, censé être neutre auparavant et, pour ce fait, produisant un ensemble de normes valides objectivement, est maintenant relié à une source subjective, sans qu'elle soit strictement individuelle; tout ce que nous nommons habituellement "sens objectif" est lié à des attitudes particulières des personnes différentes qui jugent, évaluent et interprètent cette situation de leur point de vue respectif. C'est pourquoi la normativité "globale" ou "universelle" devient soudainement localisée, fragmentée :

Une norme - nous prévient Schutz - a un certain sens "pour celui-qui-donne-lanorme (norm-giver) et pour celui-à-qui-la-norme-s'adresse (norm-addressee). Toute loi signifie quelque chose de différent pour le législateur, la personne sujette à la loi (le citoyen qui obéit la loi ou celui qui l'enfreint), la cour qui interprète la loi et I'agent qui I'applique. Le devoir a un sens différent en fonction du fait qu'il est défini par moi d'une manière autonome, ou bien qu'il me soit imposé de l'extérieur. (Schutz, 1964b, p. 276).

En jetant, génétiquement et typologiquement, un pont entre le sens objectif et subjectif, Alfred Schutz a lancé une provocation au point de vue du scientifique (du social) supposé être objectif (impartial), aussi qu'à toute forme d'objectivité censée être une pertinence imposée. Il a dévoilé non seulement les opérations constitutives de la subjectivité mondaine en tant que sens structuré socialement, mais aussi la sphère de la vie sociale intersubjective où les perti- 
nences imposées et intrinsèques sont continuellement transformées les unes dans les autres ${ }^{1}$.

Sa thèse de base est que le monde de la vie de l'individu dans l'âge moderne n'est pas complètement compréhensible, ni pour l'individu lui-même, ni pour ses semblables. Les motifs de son action sont au-delà de ses moyens. Cette situation pousse l'individu à poursuivre un travail de clarification interne, qui nécessairement le conduit à mettre en question les motifs de l'action des autres, ensemble avec les pertinences que ceux-ci imposent sur lui-même. Une analyse plus profonde déboucherait sur l'investigation de la sphère passive de l'expérience et sur les façons dans lesquelles les pertinences peuvent être imposées. Eu égard à la réalité immanente de la vie de l'acteur, elle est éminemment pratique. "Même le niveau le plus profond du flux de la conscience de l'ego solitaire, que le coup d'oeil réflexif peut pénétrer, est pramatiquement déterminé", écrit Schutz $(1932,74)$.

Cela présente des conséquences pour la validité épistémique interne de I'ego. Le scientifique du social, tout aussi comme I'homme ordinaire, met sous observation des phénomènes censés être naturels ou objectifs, c'est-à-dire non-fondés dans une constitution subjective. En conséquence, leur validité reste non-interrogée, elle va de soi (taken for granted), qu'elle soit naturelle ou objective. L'approche du phénoménologue consiste dans le choix réflexif d'un point de vue subjectif - dans le cas de Schutz: le point de vue de l'acteur social. Il configure le phénomène donné dans sa typicalité en tant qu'événement social signifiant. Il est montré alors qu'en possédant un sens pratique, l'action ou la série d'actions, ensemble avec leurs motifs et justifications, puissent être à tout moment soumises à l'inspection, à l'approbation ou au rejet, par ceux qui les observent ou par les acteurs eux-mêmes.

À cette phase de notre argumentation, il vaut la peine d'examiner brièvement comment Schutz envisage I'interaction entre les validités épistémiques et pratiques. Premièrement, il rejete "I'analogie entre I'investigateur scientifique et I'acteur des activités pratiques ordinaires" (Schutz 1943, 7)². À partir d'une

\footnotetext{
1 "Ils-relations qui sont dès le début caractérisées par un relativement bas degré d'anonymité" - écrit Schutz - "peuvent être transformées par le biais de différentes phases transitionnelles en Nousrelations." (Schutz 1932, p. 56).

2 Schutz ajoute : "Le geste initial est celui de concevoir l'acteur qui est en train de connaître les faits de la situation dans laquelle il agit et, par cela, les conditions nécessaires et les moyens disponibles pour la réalisation de ses fins. Par l'application à la relation moyens-fin, cela ressort essentielement à une question de prédiction correcte de différentes voies possibles de changer la situation (usage des moyens
} 
comparaison avec Parsons, Schutz souligne et développe la particularité du niveau théorétique en contraste avec d'autres couches de notre expérience du monde social. Il n'entame pas la description avec une définition pré-fabriquée de la rationalité. En revanche, il décrit l'agir et la pensée de l'homme ordinaire, qui vit naïvement dans le monde social parmi ses semblables. On peut observer comment, par un simple geste, Schutz loge le problème de la rationalité dans I'interaction entre le point de vue subjectif du chercheur et celui de l'homme ordinaire. Cela lui permet de faire voir que les catégories de l'interprétation du sens et les schèmes conceptuels de l'action changent dramatiquement au cours du passage d'un point de vue à un autre, d'un niveau à un autre (Idem, 8) "Avec le changement survenu dans notre point de vue - écrit Schutz - de nouveaux problèmes et des aspects factuels émergent, tandis que d'autres disparaissent, même s'ils étaient auparavant au centre de notre intérêt thématique. Ce fait seul suffit à initier une modification rigureuse du sens de tous les termes utilisés au niveau précédent. En conséquence, le contrôle attentif de ces modifications du sens s'avère être indispensable afin d'éviter le danger de transposer naïvement termes et propositions d'un niveau à un autre, même si leur validité est essentiellement confinée à un certain niveau et à ses suppositions impliquées" (Idem. p. 11). Schutz introduit ici une corrélation "contrôlée" entre les validités épistémiques et les intérêts pratiques correspondants aux points de vue et il ouvre la voie vers l'investigation phénoménologique ${ }^{3} \mathrm{du}$ "phénomène de la modification" (Ibid.) lui-même.

La description de la connaissance dans le monde de la vie ordinaire dévoile une forme de rationalité qui nous offre des voies pratiques mais consistantes de prendre une décision. Mais avant cela, nous allons analyser les ingrédients basiques de la situation de connaissance dans laquelle l'acteur se trouve luimême. Son expérience "embrasse les genres les plus hétérogènes de connais-

alternatifs) et le choix résultant d'elles. À part les questions concernant le choix des fins et celles concernant "I'effort", où le standard est à peine applicable, il y a peu de difficulté lorsqu'on conçoit l'acteur d'une manière analogue au scientiste, dont la connaissance est le déterminant principal de son action dans la mesure où sa réalisation concrète se conforme aux attentes de l'observateur qui a, comme le dit Pareto, 'une connaissance plus étendue des circonstances'.

À partir de ces principes, Parsons a développé sa théorie de "I'acte unité rational" qui est décrit comme "une unité concrète des systèmes concrets d'action. C'est une unité à laquelle on arrive, à l'intérieur du cadre du schème de l'action générale, par la maximisation d'une propriété importante des actes unité la rationalité" (1943, 8-9).

${ }^{3}$ Dans le papier en discussion, il préfère néanmoins utiliser la version plus accessible d'une théorie des modifications subjectives, à savoir celle de William James. 
sance dans un état désordonné et confus. [...] Il y a partout des brèches, intermissions, discontinuités. [...] Nous n'avons aucunement une garantie pour la sûreté de toutes les assomptions qui gouvernent notre comportement. [...] Nous ne sommes pas intérêssés de la "quête de la certitude". Nous sommes satisfaits d'avoir une chance correcte de réaliser notre but sous la main" (Idem., 15). Ce n'est pas surprénant alors, que "I'idéal de la connaissance de tous les jours n'est pas ni la certitude, ni la probabilité dans un sens mathématique mais seulement la vraisemblence. (Ibid.) Schutz n'envisage pas ici d'éveiller la rationalité idéale-formale, mais de remplacer la naturalité qu'elle présuppose et renforce continuellement lorsqu'elle est appliquée aux relations sociales et à la typicalité des actions. Ce qui est essentiel dans ce changement est que la typicalité peut perdre son apparence de naturalité et être questionnée et même changée dans un moment de réflexion.

Les anticipations des états de choses futurs sont des conjectures sur ce qui est à espérer ou à craindre ou au mieux sur ce qu'on peut raisonnablement atteindre. Plus tard, lorsque les états de choses anticipés ont pris forme en actualité, nous ne disons pas que nos prédictions sont devenues vraies ou ont été prouvées fausses, ou que notre hypothèse a été mise à l'épreuve avec succès, mais que nos espoirs ont été ou n'ont pas été bien-fondés. La consistance de ce système de connaissance n'est pas celle de la loi naturelle, mais celle des séquences typiques et des relations (Ibid.)

Par l'introduction du motif pratique dans le raisonnement, Schutz souligne les coordonnées basiques d'une pratique de la rationalité. Elles incluent la raisonabilité, la délibération, le projet (planning), la prédiction, la vraisemblance et le choix parmi deux ou plusieurs moyens (Idem, 16-20). Mais la plus importante pré-condition de cette pratique est la substitution, c'est-à-dire la capacité de l'individu de "se décider (make up his mind) et de se remplacer lui-même comme centre de son monde par un autre être animé [...]" (Idem., 20) .

La phénoménologie de Schutz a la capacité de rendre les lois, normes, ordonnances, etc. plus "familières". Si nous développons l'idée de Schutz à l'égard de la réciprocité des perspectives, nous découvrons que les normes et

\footnotetext{
${ }^{4}$ Dans ce texte Schutz se réfère lui-même au type idéal de "I'observateur », mais en anticipant la deuxième section de mon papier, je remarque que nous pouvons facillement le remplacer par toute autre personne qui incarne un point de vue "objectif » (un juge, par exemple).
} 
les lois sont plus que leur sens objectif ne l'indique, qu'elles sont aussi des perspectives subjectives. Pour Schutz, la liberté consiste dans la possibilité d'arracher notre masque, de laisser tomber le rôle, en ré-ordonnant nos orientations dans le monde social. Il nous invite de continuer à être sujets, c'est-àdire des centres d'activités spontanées, et acteurs (Schutz 1943, 20-21).

Schutz met au jour le niveau pratique des échanges entre individus. De même, il nous empêche d'accepter des situations et des événements qui nous sont imposés comme pertinents, alors qu'ils ne sont pas connectés aux intérêts choisis par nous, qu'ils ne puisent pas dans des actes à notre discrétion, et qu'ils doivent être pris tels qu'ils sont, sans que nous ayons le pouvoir de les modifier par le biais de nos activités spontanées, sauf par la transformation de pertinences imposées dans des pertinences intrinsèques. Tandis que cela reste inaccompli, nous ne considérons pas les pertinences imposées comme étant connectées à nos fins choisies spontanément. Parce qu'elles nous sont imposées, elles restent "non-clarifiées et plutôt incompréhensibles" (Schutz 1946, 127).

Schutz argumente d'une manière convaincante que les pertinences imposées se révèlent elles-mêmes dans une analyse phénoménologique en tant que sédiments des actes d'expérience précendents - mes propres actes, ainsi que ceux des autres - qui sont socialement approuvées. Ce n'est pas la rigueur de la rationalité formelle qui façonne le destin de I'homme dans les temps modernes, mais bien le tournant vers ses pouvoirs subjectifs et l'accroissement de sa capacité de prendre des décisions raisonables.

\section{LA RECONSTRUCTION PRATIQUE-HERMENEUTIQUE DE LA LOI}

La loi généralise, réduit, mesure, et fonctionne dans un cadre binaire de culpabilité et innocence. Il lui manque la patience et les ressources pour impliquer (engage) dans son réflexion les nuances des particuliers concrets (Smith, $2009,2)$. Dès le début, la phénoménologie a adopté une attitude critique envers des formes variées de positivisme et de leurs expressions dans la vie moderne. (Ibid.) Pourtant, l'approche phénoménologique a été jusqu'à maintenant minée par une ambivalence : d'une part, elle reconnaît le caractère de sens subjectif du monde social "objectif" et fournit les prémises pour une critique de 
toute sorte de formalisme et instrumentalisme ; d'autre part, elle suit l'intérêt dans une région de la constitution (pré-réflexive) des objets sociaux qui se pose elle-même comme prévalente normativement et finalement non-questionable. Par le biais d'un possible blocage du processus constitutif des impératifs dans la vie sociale, la phénoménologie n'a pas offert des garanties suffisantes ni contre le décisionisme, ni contre le conformisme moral ou légal. Par conséquent, elle n'a pas été efficace en ce qui concerne la tâche de résoudre la crise de sens qui a formé I'arrière-plan de la pratique répandue du positivisme qu'on peut rencontrer dans plusieurs apects de la vie moderne, spécialement dans des pratiques légales et politiques.

C'est pourquoi aujourd'hui la tâche de la phénoménologie devrait être encadrée dans les termes de sa possible contribution à la reconstruction du sens de la loi dans la société moderne. L'auteur mentionné plus haut dresse la carte de quelques lieux où la philosophie continentale rencontre la théorie et la pratique de la loi, parmi lesquels les plus importantes sont la réflexion sur des particuliers concrèts et les théories de l'altérité. Je pense, pourtant, que les noeuds par où la phénoménologie peut infiltrer et transformer des pratiques de la loi et légales sont encore très largement ignorés. Il me semble que la phénoménologie comme pratique analytique soit particulièrement bien douée pour secouer l'apparence de la conviction absolue qui règne sur toute sorte de procédures impersonnelles, dont celles judiciaires sont exemplaires. Spécialement, I'originale phénoménologie du comportement (social) humain d'Alfred Schutz semble bien adaptée à la tâche de décrire des pratiques dans une société déterminée et d'adresser ainsi les conséquences pratiques des actions dans une manière qui les rende transparente aux acteurs sociaux et susceptible de changement.

Dans la société moderne, les activités professionnelles sont dévenues hautement spécialisées et codifiées et leur contenu consiste au fond dans des règles explicites appliquées de façon impersonnelle. La montée de l'efficacité bureaucratique a transformé la vie professionnelle dans une série d'actes quasimécaniques et tend à éliminer toute référence à ce que Max Weber a appelé "la rationalité-valeur". En conséquence, elle tend à évacuer toute référence aux valeurs personnelles ou substantielles que le professionnel assume. Probablement, il n'y a aucune autre profession que celle légale où le positivisme soit dévenu une doctrine à part entière et une sorte d'idéologie professionnelle ; il 
s'est donc profondément incrusté dans les pratiques courantes. ${ }^{5}$ Un diagnostic du judiciaire dans les sociétés contemporaines démocratiques ou engagées dans un processus de démocratisation ne peut faire autre chose que présenter une énorme série de symptômes négatifs, allant de contraintes formelles inefficaces de l'État administratif jusqu'aux pressions informelles et illégitimes. Formalisme, légalisme (la loi en tant que dépôt autonome de principes justes) et la rhétorique morale sont largement répandus, pour n'en mentionner l'ennui et la bureaucratie comme traits du sytème qui sont encore tous très proéminents ${ }^{6}$.

Est-il toujours possible d'induire quelques "valeurs" de base dans un système adminstratif aux allures d'une machine bureaucratique? Peut-on imaginer les lois autrement que comme mûrs et barrières? Est-ce que la loi peut procéder d'une façon qui fasse place aux valeurs morales véritables? La phénoménologie peut répondre affirmativement à ces questions par une réconfiguration du champ des pratiques normatives de telle sorte qu'elle limite les effets et I'influence du positivisme.

La non-coïncidence du subjectif-objectif, tout aussi que la dialectique subjacente et compliquée du sens subjectif et objectif, non seulement serve à réencadrer la question de principes de la normativité, mais, de même, mais aussi à surmonter le positivisme légal et politique. La tradition positiviste fait preuve d'aversion motivée politiquement envers toute investigation concernant la nature "subjective" et envers les voies spécifiques du fonctionnement de la loi. À partir des anées 1960, I'explication de la décision des juges a pris une place centrale dans la description des voies du fonctionnement de la loi et a été intégrée dans la théorie générale de la normativité. La nouvelle question de base était si les juges devraient adopter une compréhension vide, instrumentale de la légalité et de l'autorité de la loi ou bien une vision de principe sur la légalité. Les pratiques normatives contemporaines, spécialement celles légales, s'efforcent d'intégrer à la fois les valeurs et les fins afin de faire avec les exigences d'une société multi-différenciée. Dorénavant, la loi ne peut plus être visée en tant qu'objet en lui-même, en tant qu'objet purement épistémique; elle est un objet qui se définit lui-même par se conséquences pratiques, tout

\footnotetext{
${ }^{5}$ Une discussion sur le positivisme pratique dans les professions légales, peut être trouvée in Copoeru 2008a.

${ }^{6}$ Pour une analyse du judiciaire dans le contexte de la transition démocratique dans la Roumanie post1989, voir Copoeru 2008b.
} 
comme par les manières du raisonnement et par les types de rationalité qu'elle présuppose lorsqu'elle est mise au travail. Quand nous nous occupons de normes et de lois, nous devrions renoncer à la perspective qui consiste dans I'application des règles aux faits, et nous devrions en adopter une autre, caractérisée par la prise de décision et par la quête de possibles solutions. Cela entraîne un changement important dans le sens de la loi, à savoir un changement du sens épistémique de la loi avec son sens pragmatique.

Mais comment est-il possible d'intégrer dans le noyau du raisonnement légal (normatif) des références à des fins et buts sans porter préjudice à l'objectivité du raisonnement lui-même? Et est-ce qu'il est possible d'intégrer dans la structure de la loi quelque chose qui lui soit extérieur sans démonter la loi elle-même?

Selon l'approche shutzienne, les lois ont non seulement un sens objectif, mais primairement elles sont des perspectives subjectives. La phénoménologie de Schutz a sans doute la capacité de rendre les lois, normes, ordonnances, etc., plus "familières". Mais, elles sont en même temps le produit d'un sujet, plus précisément, d'un sujet libre en tant que centre d'activité spontannée et en tant qu'acteur social (Schutz 1996a, p. 20-21).

Le juge fait face souvent à l'abysse des cadres hétérogènes. Par exemple, est-ce que le sentiment que la règle légale "devrait" être interprétée d'une manière particulière a son origine dans nos intuitions à l'égard de "I'intention" de ceux qui l'ont l'écrit ou bien dans la structure de la conscience légale? (Boyle, 1991) Il n'y pas d'autre façon de résoudre ce problème que si nous le considérons comme un problème pratique. Dans l'empire pratique nous ne trouvons jamais en manque de possibilités. En praticiens, dans le pire des scénarios, nous pouvons simplement suivre la routine. Or, le juge peut adopter une forme de rationalité pratique ou provisoire et peut renoncer à l'idée de juger sur la base de schèmes universaux, non-questionables. Quoi qu'il en soit, selon Schutz, le transfert des schèmes de la rationalité formelle qui appartiennent aux autres champs de connaissance vers les sphères de la décision pratique semble être la pire de toutes les solutions possibles. 


\section{CONCLUSIONS}

J'ai essayé d'argumenter dans ce papier que l'introduction par Schutz d'un motif pragmatique dans l'analyse phénoménologique de la conscience, ensemble avec la dialectique du sens subjectif et objectif (imposé), sont à même de dévoiler une dimension originelle de la normativité, qui nous permet de reconstruire le concept de la loi.

J'ai posé la question sur comment l'approche phénoménologique schutzienne envers la normativité et la rationalité, présentée sommairement dans ce papier, peut s'avérer utile dans la reconstruction du sens de la loi.

Alfred Schutz n'a jamais été dépassé en ce qui concerne ses efforts analytiques de mettre au jour le rôle du sens "subjectif" dans le monde socioculturel. Il nous offre des modalités non seulement de comprendre ce que signifie une pratique commune de la construction du sens social, mais aussi de la provoquer de l'intérieur du point de vue des opérations "subjectives". Ainsi, la dé-naturalisation des phénomènes sociaux, que Schutz réalise avec succès, fournit au chercheur les outils nécessaires à aborder les pratiques légales et les fictions correspondantes qu'elles ont engendré. Le discours de la loi doit être complété non seulement par une phénoménologie critique, mais aussi par une phénoménologie reconstructive.

Le geste de donner à la subjectivité (transcendentale ou non) une place centrale dans la reconstruction phénoménologique constitutive des pratiques légales nous conduit à la réconnaissance "d'un doute au sein du sens" (Kennedy $1997,177)$ et nous permet de considérer le problème de la dédifférentiation (Schlag 2009) et du positivisme pratique dans le monde moderne.

\section{BIBLIOGRAPHIE}

BARBER, M. (2004): The Participating Citizen: A Biography of Alfred Schutz. Albany: State University of New York Press.

BOYLE, J. "Is Subjectivity Possible - The Post-Modern Subject in Legal Theory" (1991). Duke Law Faculty Scholarship. Paper 620.

COPOERU, I. (2008a): "A Schutzian Perspective on the Phenomenology of Law in the Context of Positivistic Practices." In Human Studies 31/3. (2008), pp. 269-277 
- (2008b). "Gibt es Raum für Ethik? Implementation richterlicher und staatsanwaltschaftlicher Diskursethik in Rumänien." In Kritische Vierteljahresschrift für Gesetzgebung und Rechtswissenschaft. 4/2008, pp. 468-474

GABEL, P. (1980): "Reification in Legal Reasoning", Research in Law and Sociology, vol. 3, pp. 25-51 (Toronto: University Press)

- (1984). The Phenomenology of Rights-Consciousness and the Past of the Withdrawn Selves, Texas Law Review, pp. 1563-1577

KENNEDY, ennedy, D. (1997): A Critique of Adjudication (fin de siècle). Harvard University Press, 1997

KRESS, K (1992): "Legal Indeterminacy and Legitimacy", in: Gregory Leyh (Ed.), Legal hermeneutics: history, theory, and practice, University of California Press, Berkeley and Los Angeles, 1992, pp. 200-218

LEYH, G. (2009): Legal Education and the Public Life, in: Gregory Leyh (Ed.), Legal hermeneutics: history, theory, and practice, University of California Press, Berkeley and Los Angeles, 1992, pp. 269-298

SCHLAG, P. (2009): "The dedifferentiation problem." In Continental Philosophy Review, (2009) 42, pp. 35-62

SCHUTZ, A. (1928): "Toward a Viable Sociology." In Alfred Schutz, Collected Papers, vol. IV, H. Wagner, G. Psathas, \& F. Kersten (Eds.), Dordrecht: Kluwer Academic Publishers, 76-83

- (1932): "The dimensions of the social world". In Alfred Schutz, Collected Papers, vol. II, A. Brodersen (Ed.), (pp. 20-62). The Hague: Martinus Nijhoff

- (1943): "The Problem of Rationality in the Social World. A Lecture Delivered at the Faculty Club of Harvard University on April 13th/1940." In Alfred Schutz, Collected Papers, vol. IV, H. Wagner, G. Psathas, \& F. Kersten (Eds.), Dordrecht: Kluwer Academic Publishers, 6-24

- (1946): "The Well-Informed Citizen: An Essay on the Social Distribution of Knowledge" In Alfred Schutz, Collected Papers, vol. II, Studies in Social Theory, edited by A. Brodersen, The Hague: Martinus Nijhoff, 1964

- (1952): "Gnosticism and Orthodoxy: Contrasts in Fundamental Metaphysical and Theological Positions." In Alfred Schutz, Collected Papers, vol. IV, H. Wagner, G. Psathas, \& F. Kersten (Eds.), Dordrecht: Kluwer Academic Publishers, 221-233

- (1964a) "Equality and the meaning structure of the social world." In Alfred Schutz, collected papers, vol. II: Studies in social theory. A. Brodersen (Ed.),. The Hague: Martinus Nijhoff, 226-273

- (1964b): "Some equivocations in the notion of responsibility." In Alfred Schutz, collected papers, vol. II: Studies in social theory. A. Brodersen (Ed.), The Hague: Martinus Nijhoff, 274-276

- (1978): "Parsons' theory of social action: A critical review by Alfred Schutz." In Theory of Social Action, R. H. Grathoff (Ed.), Bloomington: Indiana University Press, 1978, 8-60

SMITH, N. (2009): "Introduction." In Continental Philosophy Review, (2009) 42:1-4. 